\title{
Competências gerais e habilidades específicas: a realidade do ciclo básico
}

Pedro Henrique Sette-de-Souza*; Leonardo Cesar Amaro-da-Silva**; Lidya Nara Marques de Araújo $^{* * *}$; Lucas José de Azevedo Silva ${ }^{* * *}$; Olga Benário Vieira Maranhão ${ }^{* * * *}$; Isabelita Duarte de Azeve$\mathrm{do}^{* * * * *}$

* Professor da Universidade Estadual da Paraíba

** Cirurgião-Dentista graduado pela Universidade Federal do Rio Grande do Norte

**** Estudante do Curso de Odontologia da Universidade Federal do Rio Grande do Norte

Cirurgiã-Dentista graduada pela Universidade Federal do Rio Grande do Norte

Professora da Universidade Federal do Rio Grande do Norte

\section{RESUMO}

A formação em odontologia vem, ao longo dos anos, sofrendo significativas mudanças. A última alteração considerável foi a construção e implementação das Diretrizes Curriculares Nacionais (DCN), em 2002, para os cursos de graduação. Visando formar cirurgiões-dentistas dotados de conhecimento e pensamento crítico, reflexivo e humanístico, as DCN vêm para guiar a formação profissional para qualquer mercado de trabalho. Assim, esse estudo visou avaliar e discutir a estrutura curricular dos primeiros quatro períodos do curso de Odontologia da Universidade Federal do Rio Grande do Norte com base nas competências gerais e habilidades específicas propostas pelas DCN. Foi realizada a categorização das Habilidades Específicas tomando como base as competências gerais. Em seguida, com base nas categorias formadas, foram detectados os momentos nesses dois anos iniciais em que as mesmas eram desenvolvidas. Ao contrário do que se pensava, a primeira metade do curso, tida como "ciclo básico", que abrange as disciplinas comuns a toda a área da saúde, trabalha elementos importantes das DCN e ainda traz a perspectiva de educação permanente, uma das competências gerais para o futuro profissional. Conclui-se que o "ciclo básico" do curso de odontologia da UFRN contempla todas as competências gerais e habilidades específicas propostas pelas DCN para o curso de Odontologia.

Descritores: Educação em Odontologia. Ensino. Educação Superior.

\section{INTRODUÇÃO}

O curso de formação em odontologia da Universidade Federal do Rio Grande do Norte prioriza a formação de um Cirurgião-Dentista generalista, humanista, de habilidade crítica e reflexiva, de maneira a atuar em todos os níveis de atenção à saúde, com base no rigor técnico e científico ${ }^{1}$, trazendo benefício à sociedade por meio de transformação favorável no que diz respeito ao fazer saúde. Tais princípios corroboram com as Diretrizes Curriculares Nacionais (DCN) para os cursos de graduação em Odontologia.

Deve ser ressaltado que as DCN assumem um papel estratégico no aperfeiçoamento do Sistema Único de Saúde (SUS). Ao mesmo tempo, a discussão sai dos limites acadêmicos à medida que a sociedade -15(1): 38-47, 2015. 
começa a exigir melhores serviços de saú$\mathrm{de}^{2}$. Assim, o SUS precisa ser entendido como um interlocutor essencial das escolas na formulação e implementação dos projetos pedagógicos de formação profissional e não mero campo de estágio ou aprendizagem prática. Esta articulação é reforçada no objeto das DCN quando visa "Construir perfil acadêmico e profissional com competências, habilidades e conteúdos contemporâneos, bem como, para atuarem, com qualidade e resolutividade, no Sistema Único de Saúde"3.

Dessa maneira, as DCN acabam por corresponsabilizar as Instituições de Ensino Superior (IES) na escolha de qual referencial devem adotar para construir o seu currículo ${ }^{4}$. Torna-se interessante a elaboração do Projeto Pedagógico respeitando-se o diagnóstico do perfil, das expectativas e necessidades dos discentes e das necessidades da população quanto à saúde bucal; a definição de metas e objetivos que se pretendem alcançar e o estabelecimento de diretrizes gerais que nortearão o curso; planejar ações que permitam atingir as metas desejadas; organizar o processo de acompanhamento e avaliação do projeto para seguir o desencadeamento das ações, perceber seus resultados e redirecionar os rumos, quando necessário ${ }^{5}$.

Considerando que as práticas odontológicas e o seu ensino formal existem na sociedade e para o conjunto da sociedade, devendo ser pensadas a partir da pluralidade e da conjugação política de interesses públicos que precisam ser vistos e debatidos por todos os segmentos sociais interessados, deve ser reconhecida a formação universitária em íntima relação com o SUS. Logo, a filosofia e organização dos serviços desse sistema deve ser consolidada por pro- fissionais com determinadas competências e habilidades. De modo a possibilitar opções conscientes e embasadas em fundamentos científicos, no conhecimento crítico e baseado na experiência concreta da realidade social, consolidado pela aproximação entre a universidade e o SUS ${ }^{6}$.

Os estudos relacionados à graduação em odontologia no Brasil apresentam convergência com relação às suas propostas de enfrentamento dos problemas, ou seja, a ressignificação do papel da universidade, do ensino de graduação na formação de profissionais de saúde. A construção/implementação das DCN para os cursos de graduação em odontologia no Brasil está associada, de um lado, à reestruturação produtiva do capitalismo global, particularmente à acumulação flexível e à flexibilização do trabalho. Por outro lado, se insere na luta da universidade pela reconquista de sua legitimidade social e pela consolidação do SUS em consonância com as necessidades sociais da maioria da população brasileira $^{7}$.

O planejamento do curso de graduação de odontologia deve se fundamentar no projeto pedagógico, proposta de trabalho integrado que descreve um conjunto de capacidades a serem desenvolvidas pelos alunos $^{5}$. Os discentes têm um papel importante na elaboração e discussão desse projeto pedagógico, em decorrência de estar diretamente em contato com os conteúdos e metodologias adotadas para sua formação. Ao entrarem em contato com a realidade da população, notarão uma significativa diferença social e econômica quando comparado ao seu meio, o que exigirá um conhecimento que vai além do adquirido na sala de aula até então; o que torna necessária a busca de soluções ${ }^{8}$. 
Com base na tradução dos conhecimentos e habilidades em ações práticas no campo de trabalho durante a formação dos graduandos, denota-se de maneira objetiva a aplicabilidade das disciplinas prestadas no campo prático. Sabe-se que na estrutura curricular do curso da referida instituição, seguindo um padrão, são determinadas disciplinas de cunho de conhecimento geral (referentes à área da saúde, entendido como ciclo básico), que precedem disciplinas de cunho de conhecimentos específicos (que possibilitam o aprendizado técnicocientífico pelo estudante). Deve-se fazer uso de metodologias de ensinoaprendizagem que permitam a participação ativa dos alunos neste processo e a integração dos conhecimentos das ciências básicas, sociais e clínicas. De maneira que, ao final, devem prestar contribuição direta no desenvolvimento das capacidades requisitadas do futuro cirurgião-dentista ${ }^{5}$.

Diante disto, surge a necessidade de analisar e discutir a estrutura curricular do curso de Odontologia da Universidade Federal do Rio Grande do Norte (UFRN) sob a perspectiva dos discentes, a fim de debater a atual situação de maneira a lançar olhar sobre as disciplinas ofertadas desde o início do curso até o conteúdo específico, verificando a incumbência das disciplinas no desenvolvimento das habilidades específicas do graduando, com base nas DCN e no perfil formador da instituição. Desta forma é possível sinalizar possíveis pontos negativos e/ou sujeitos à modificação, passíveis de reformulação.

\section{METODOLOGIA}

O Grupo Estudantil de Debates Pedagógicos (GEDePe), que conta com acadêmicos de todos os períodos do curso, foi criado por iniciativa dos estudantes da UFRN para discutir assuntos relacionados ao ensino de odontologia. Inicialmente, os graduandos categorizaram as habilidades específicas propostas pelas DCN para os cursos de odontologia ${ }^{3}$, utilizando como grande grupo (ou categoria) as seis competências gerais. Tal categorização obedeceu à descrição de cada competência geral e habilidade específica, sendo feita, portanto, por aproximação de significado, através de uma análise crítica-reflexiva ${ }^{9}$. Em um segundo momento, na estrutura curricular do curso da UFRN vigente no ano de 2013, foram identificadas quais habilidades específicas (e consequentemente, qual competência geral) eram trabalhadas em cada uma das disciplinas obrigatórias dos quatro primeiros períodos do curso ( $1^{\circ}$ e $2^{\circ}$ ano).

\section{RESULTADOS}

Os resultados são apresentados nos quadros 1 e 2, em relação à categorização das DCN de Odontologia: habilidades específicas segundo competências gerais e localizando das competências gerais e habilidades específicas nas disciplinas do "ciclo básico”, respectivamente. 
Quadro 1 - Categorização das DCN de Odontologia: habilidades específicas segundo competências gerais

\section{Atenção à} Saúde

Atuar em todos os níveis de atenção à saúde, integrando-

se em programas de promo-

ção, manutenção, preven-

ção, proteção e recuperação

da saúde, sensibilizados e

comprometidos com o ser

humano, respeitando-o e

valorizando-o (AS1)

Atuar multiprofissionalmen-

te, inter e transdisciplinar-

mente com extrema produ-

tividade na promoção da

saúde baseado na convicção

científica, de cidadania e de

ética (AS2)

Reconhecer a saúde como

direito e condições dignas

de vida e atuar de forma a

garantir a integralidade da

assistência, entendida como

conjunto articulado e contí-

nuo das ações e serviços

preventivos e curativos,

individuais e coletivos,

exigidos para cada caso em

todos os níveis de comple-

xidade do sistema (AS3) (continua)

Tomada de Decisões Comunicação Ciderança

Administração Gerenciamento

Educação Permanente cas e procedimentos opera- efetivamente com paci- de forma articulada ao tórios (TD1) entes, trabalhadores da área da saúde e outros indivíduos relevantes, grupos e organizações (CO1)

contexto social, en-

tendendo-a como uma

forma de participação

e contribuição social

(LD1)

Obter e eficientemente gra- Manter reconhecido

var informações confiáveis e padrão de ética profis-

avalia-las objetivamente

sional e conduta, e

aplicá-lo em todos os

aspectos da vida profissional (CO2)

\section{Estar ciente das regras}

dos trabalhadores da

área da saúde bucal na

sociedade e ter res-

ponsabilidade pessoal para com tais regras (LD2)

Aplicar conhecimentos de

Comunicar-se com

Trabalhar em equipes saúde bucal, de doenças e pacientes, com profistópicos relacionados no sionais da saúde e com lhor interesse do indiví-

a comunidade em gera

interdisciplinares e atuar como agente de

promoção de saúde duo e da comunidade (TD3)
(LD3)

\section{(AG3)}

\section{Respeitar os princí-} pios éticos e legais inerentes ao exercício eficientemente (AG2) componente da obri-
Organizar, manusear e

avaliar recursos de cui-

dados de saúde efetiva e pretar dados para a construção do diagnóstico profissional (EP1)

epidemiológicas e clíni-

Conhecer métodos e técnicas de investigação e elaboração de trabalhos acadêmicos e científicos (EP2)

Participar em educação continuada relativa a saúde bucal e doenças como um gação profissional e manter espírito crítico, mas aberto a novas informações (EP3) 
Quadro 1 - Categorização das DCN de Odontologia: habilidades específicas segundo competências gerais

\begin{tabular}{|c|c|c|c|c|c|}
\hline $\begin{array}{c}\text { Atenção à } \\
\text { Saúde }\end{array}$ & Tomada de Decisões & Comunicação & Liderança & $\begin{array}{l}\text { Administração e } \\
\text { Gerenciamento }\end{array}$ & $\begin{array}{c}\text { Educação Per- } \\
\text { manente }\end{array}$ \\
\hline $\begin{array}{l}\text { Desenvolver assistência } \\
\text { odontológica individual e } \\
\text { coletiva (AS4) }\end{array}$ & $\begin{array}{l}\text { Participar de investigações } \\
\text { científicas sobre doenças e } \\
\text { saúde bucal e estar prepara- } \\
\text { do para aplicar os resultados } \\
\text { de pesquisas para os cuida- } \\
\text { dos de saúde (TD4) }\end{array}$ & & & $\begin{array}{l}\text { Planejar e administrar } \\
\text { serviços de saúde comu- } \\
\text { nitária (AG4) }\end{array}$ & $\begin{array}{l}\text { Reconhecer suas } \\
\text { limitações e estar } \\
\text { adaptado e flexível } \\
\text { face às mudanças } \\
\text { circunstanciais (EP4) }\end{array}$ \\
\hline $\begin{array}{l}\text { Identificar em pacientes e } \\
\text { em grupos populacionais as } \\
\text { doenças e distúrbios buco- } \\
\text { maxilo-faciais e realizar } \\
\text { procedimentos adequados } \\
\text { para suas investigações, } \\
\text { prevenção, tratamento e } \\
\text { controle (AS5) }\end{array}$ & $\begin{array}{l}\text { Propor e executar planos de } \\
\text { tratamento adequados (TD5) }\end{array}$ & & & & $\begin{array}{l}\text { Acompanhar e in- } \\
\text { corporar inovações } \\
\text { tecnológicas (in- } \\
\text { formática, novos } \\
\text { materiais, biotecno- } \\
\text { logia) no exercício } \\
\text { da profissão (EP5) }\end{array}$ \\
\hline \multicolumn{6}{|l|}{$\begin{array}{l}\text { Promover a saúde bucal e } \\
\text { prevenir doenças e distúr- } \\
\text { bios bucais (AS6) }\end{array}$} \\
\hline $\begin{array}{l}\text { Realizar a preservação da } \\
\text { saúde bucal (AS7) }\end{array}$ & & & & & \\
\hline
\end{tabular}


Quadro 2 - Localização das competências gerais e habilidades específicas nas disciplinas do “ciclo básico” do Curso de Odontologia da UFRN

(continua)

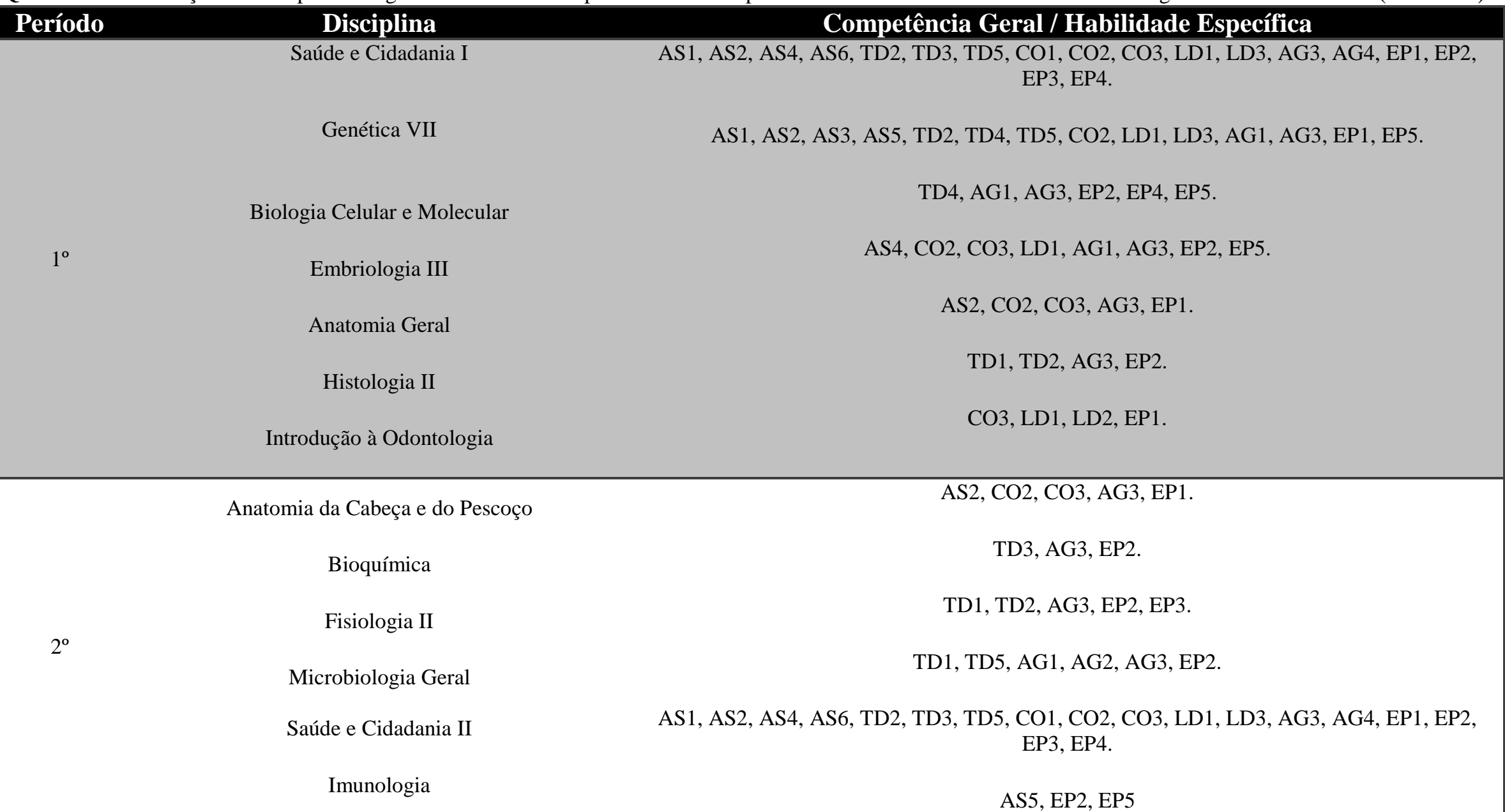


Quadro 2 - Localização das competências gerais e habilidades específicas nas disciplinas do “ciclo básico” do Curso de Odontologia da UFRN

\section{Disciplina}

Farmacologia II

Patologia Geral

Metodologia da Pesquisa Científica

Odontologia, Cultura e Sociedade

Microbiologia e Imunologia Oral

Anatomia e Escultura Dentária

Psicologia Aplicada a Odontologia

Saúde Bucal Coletiva I

Patologia Oral

Estomatologia

$4^{\circ}$
Imagenologia

Cariologia

Materiais Dentários
Competência Geral / Habilidade Específica

AS3, AS4, TD3, TD5, AG3, EP5.

AS3, CO1, CO3, EP2, EP5.

TD1, TD2, CO1, CO2, LD1, AG1, AG3, EP1, EP2.

AS1, AS2, AS3, AS6, AS7, TD3, TD5, CO1, CO2, CO3, LD1, LD2, EP1, EP2.

AS1, AS5, AS6, AS7, TD2, TD3, TD4, AG1, AG2.

AG1, EP5.

CO1, LD3, EP1.

AS1, AS3, AS4, AS5, AS6, TD1, TD2, TD3, TD4, LD1, AG1, AG2, AG3, EP2.

AS5, AS7, TD2, AG3.

AS1, AS3, AS4, AS5, AS6, AS7, TD1, TD2, TD3, TD5, CO1, CO3, AG1, AG3, EP1, EP2, EP5.

TD2, TD5, CO1, CO3, AG3, EP5.

AS7, TD5, EP3, EP5.

AG1, AG3, EP2.

AS1, AS2, AS3, AS4, AS5, AS6, AS7, TD1, TD2, TD3, TD4, TD5, CO1, CO2, CO3, LD1, LD2, LD3, AG1, AG2, AG3, EP1, EP2, EP3,EP4, EP5. 


\section{DISCUSSÃO}

Ao considerar o entendimento e a experiência do acadêmico da odontologia, busca-se ampliar o conhecimento do cirurgião dentista e torná-lo capaz de atender efetivamente uma maior parcela da população. Ainda é válido ressaltar que a visão discente sobre o projeto pedagógico do seu curso é de extrema importância, pois é ele quem realmente vive as dificuldades e entende o currículo eventualmente até melhor do que grande parte dos que o elaboram. Seu posicionamento sobre como as DCN são trabalhadas no curso reflete a clareza com a qual as competências são estimuladas e desenvolvidas em cada conteúdo curricular.

Nesse contexto, introduzir mais disciplinas optativas, flexibilizando o currículo, pode possibilitar o aprofundamento da aprendizagem sobre determinados assuntos, o que resultaria na ampliação do conhecimento do corpo discente e possibilitaria sua atuação em diversos campos da odontologia durante a graduação. A partir dessa alteração, o discente atua como um dos sujeitos de mudança, ou seja, torna-se responsável pela construção de melhorias para seu curso, as quais serão benéficas para este grupo, para a Universidade e ainda para a população.

Outro fator que deve ser ressaltado é a provável integração (no sentindo de atingir as competências e habilidades) que permeia o “ciclo básico” da odontologia na UFRN. Todas as seis competências gerais (e cerca de $90 \%$ a $95 \%$ das habilidades específicas) são trabalhadas em cada período. Não é necessário que uma disciplina sozinha contemple todas as habilidades específicas. Contudo, é de grande valia que o conjunto de disciplinas obrigatórias de um semestre seja capaz de atingir este objetivo, como é observado no currículo em estudo.
Quando as disciplinas são analisadas separadamente, observa-se que "Saúde e Cidadania I e II" predomina no trabalho das habilidades específicas no primeiro ano do curso. Isso pode estar relacionado com o fato de ser uma das poucas atividades de todo o currículo do curso que permite ao estudante de odontologia ir à Unidade de Saúde da Família, entender a organização daquela comunidade, refletir acerca dos problemas encontrados e realizar uma intervenção local, tudo isso com uma equipe multidisciplinar composta de estudantes de vários outros cursos da Universidade. Portanto, essas disciplinas tem mais um cunho social do que clínico.

Por outro lado, a disciplina do período em questão que mais trabalha tais habilidades é a Clínica Integrada Nível I, trabalhando o atendimento clínico propriamente dito. Ao ser colocado frente a frente com o paciente, o acadêmico já precisa ter uma série de habilidades para lidar com ele, buscando a melhor forma de resolver o problema e sendo capaz de estabelecer uma boa comunicação.

Contudo, algo que precisa ser esclarecido é que a identificação Competência Geral/Habilidade Específica (quadro 2) não é 'fechada', ou seja, o estudante não terá, necessariamente, a capacidade de "desenvolver assistência odontológica individual e coletiva” após cursar uma disciplina que trabalhe esse tópico, pois, ressalta-se aqui que, os dois primeiro anos do curso não denotam totalmente o conhecimento específico da Odontologia propriamente dita, principalmente a parte assistencialista/curativa. Entretanto, a classificação nos revela que a disciplina trabalha elementos que mais tarde, em uma disciplina mais clínica, serão amadurecidos, caracterizando um nível incipien- 
te de educação continuada com níveis de complexidade crescente, dentro da própria graduação.

\section{CONCLUSÕES}

O “ciclo básico” do curso de Odontologia da UFRN atende a todas as competências gerais e habilidades específicas propostas pelas DCN. O desenvolvimento de grande parte das habilidades específicas e de todas as competências gerais durante esse período traz uma perspectiva de educação continuada já na graduação, uma vez que não há um abismo entre o que é trabalhado em um semestre e nos seguintes. A maneira pela qual as disciplinas estão organizadas no currículo consegue fazer com que o acadêmico possa estar sempre trabalhando todas as habilidades requeridas de um cirurgiãodentista desde o início da sua formação. Em qualquer processo de mudança curricular, a participação discente é de suma importância, pois são eles quem tem o real conhecimento de como está o curso, o processo ensinoaprendizado e o que/como pode melhorar.

\section{REFERÊNCIAS}

1. Centro de Ciências da Saúde. Projeto Político-Pedagógico do Curso de Odontologia da Universidade Federal do Rio Grande do Norte. UFRN. 2006.

2. Morita MC, Kriger L. Mudanças nos cursos de Odontologia e a interação com o SUS. Rev ABENO 2004;4(1):17-21.

3. Brasil. Ministério da Educação. Diretrizes Curriculares Nacionais dos Cursos de Graduação em Farmácia e Odontologia. Conselho Nacional de Educação. Diário Oficial da União, Seção 1, p. 25 7/12/2001.

4. Pereira IDF, Lages I. Diretrizes curriculares para a formação de profissionais de saúde: competências ou práxis? Trab. educ. saúde. 2013;11(2): 319-38.

5. Perri de Carvalho AC. A Odontologia em tempos da LDB. Canoas: ULBRA; 2001.

6. Silveira JLC. Diretrizes Curriculares Nacionais Para os Cursos de Graduação em Odontologia: historicidade, legalidade e legitimidade. Pesq. Bras. Odontoped. Clin. Integr. 2004;4(2): 151-6.

7. Senna MIB, Lima MLR. Diretrizes Curriculares Nacionais para o ensino de graduação em odontologia: uma análise dos artigos publicados na revista da ABENO, 2002-2006. Arq. odontol. 2009;45(1):306.

8. Mialhe FL, Melo MM, Berti M, Dobrowolski M. Contribuição das ações de educação em saúde da disciplina de odontologia em saúde coletiva na consolidação das DCN e do SUS: relato de uma experiência. Arq. Ciênc. Saúde Unipar. 2007;11(3):193-7.

9. Bardin L. Análise de Conteúdo. Lisboa: Edições 70. 2006.

\section{ABSTRACT \\ General Competencies and Specific Skills: the reality of the basic cycle}

Training in Dentistry has, over the years, undergoing significant changes. The last major change was the construction and implementation of the National Curriculum Guidelines (DCN) in 2002 for undergraduate courses. In order to form dental surgeons endowed with knowledge and critical, reflective and humanistic thoughts, DCN is to guide the training for any job market. Thus, this study aimed to evaluate and discuss the curricular structure of the first four periods of the Dental course at Federal University of Rio Grande do Norte regarding general and 
specific skills proposed by DCN. The categorization of specific skills based on general skills was conducted. Then, on the basis of these categories, the moments in the early years they were developed. Unlike previously thought, the first half of the course, considered "basic cycle" which includes common disciplines to all health courses, works important elements of DCN and also brings the perspective of permanent education, one of the general competencies for the future professional. It was concluded that the "basic cycle" of Dental School at UFRN includes all general and specific skills proposed by DCN for the dental course.

Descriptors: Dental Education. Teaching. Higher Education. Higher Education.

Correspondência para:

Pedro Henrique Sette-de-Souza

e-mail: pedro_sette_@hotmail.com

Rua Santa Tereza D'Avilla, 3322

Candelária 59065-670 - Natal/RN 